\title{
Expresión de CD10 y del marcador de carcinoma renal en el carcinoma renal de células claras: Análisis en matrices tisulares
}

\author{
J.A. Ortiz-Rey, C. Gómez De María, E. Peláez Boismorand, A. Fernández Costas, \\ M.J. Barbosa Barreiro, I. Antón Badiola.
}

Servicio de Patología. Hospital POVISA. Vigo (Pontevedra).

Actas Urol Esp 2006; 30 (3): 281-286

\section{RESUMEN}

EXPRESIÓN DE CD10 Y DEL MARCADOR DE CARCINOMA RENAL EN EL CARCINOMA RENAL DE CÉLULAS CLARAS: ANÁLISIS EN MATRICES TISULARES

Objetivos: Los anticuerpos CD10 y marcador de carcinoma renal (MCR) reaccionan frente a proteínas del epitelio normal del túbulo contorneado proximal, expresándose por tanto en los carcinomas de células renales. Se analizan la frecuencia y el patrón de expresión de ambos marcadores inmunohistoquímicos en una serie de carcinomas renales de células claras.

Método: Se han utilizado dos matrices tisulares (“tissue arrays”) constituidas por cilindros obtenidos con aguja 16G de 40 bloques de parafina correspondientes a carcinomas renales de células claras. Se realizó técnica de estreptavidina marcada - biotina (LSAB2, Dako) con anticuerpos monoclonales CD10 y MCR (Novocastra), ensayándose para este último distintos métodos de recuperación antigénica. Se valoró la positividad como + (aislada o muy focal); ++ (moderada) y +++ (extensa).

Resultados: Con CD10 fueron positivos 30 casos (75\%): 12 +; 5 ++ y 13 +++. Con respecto a MCR, el mejor método de desenmascaramiento antigénico fue un doble tratamiento enzimático (tripsina + proteasa). Se encontró positividad para MCR en 20 casos (50\%): 7+; 5 ++ y 8 +++. De los 20 casos positivos para MCR, cuatro habían sido negativos para CD10. Los otros 16 expresaban también CD10.

Conclusiones: CD10 y MCR se expresan con frecuencia en los carcinomas renales de células claras, por lo que pueden ser marcadores útiles para sugerir en un carcinoma un origen renal. MCR presenta menor sensibilidad que CD10. Al ser la expresión de ambos habitualmente focal, la sensibilidad de estas técnicas es menor cuando se utilizan muestras pequeñas, como son las matrices tisulares. En la técnica IHQ para MCR resulta crucial el método de desenmascaramiento antigénico, obteniéndose los mejores resultados con el uso de enzimas proteolíticos.

Palabras Clave: CD10. CALLA. Marcador de carcinoma de células renales. Carcinoma de células renales. Carcinoma de células claras. Inmunohistoquímica.

\section{ABSTRACT \\ EXPRESSION OF CD10 AND RENAL CELL CARCINOMA MARKER IN CLEAR CELL RENAL CELL CARCINOMA: ANALYSIS ON TISSUE ARRAYS}

Objectives: $\mathrm{CD} 10$ and renal cell carcinoma (RCC) marker antibodies react against proteins of the epithelium of the renal proximal tubule, being expressed by renal cell carcinomas. The frequence and pattern of expression of both markers are analysed in a series of clear cell renal cell carcinomas.

Method: Two tissue arrays were used, which were composed of cylinders obtained with a $16 \mathrm{G}$ needle from 40 paraffin blocks that corresponded to clear cell renal cell carcinomas. The labeled streptavidin-biotin technique was performed (LSAB2, Dako) using CD10 and RCC monoclonal antibodies (Novocastra), testing different antigen retrieval methods for RCC. Immunoreactivity was evaluated as + (isolated cells or focal staining); ++ (moderate) and +++ (extense).

Results: Thirty cases (75\%) were positive for CD10: $12+; 5++$ and $13+++$. The best antigen retrieval method for RCC was a double enzyme digestion (trypsin + protease). Twenty cases $(50 \%)$ were positive for RCC: $7+; 5++$ and $8+++$ Four cases out of the 20 immunoreactive for RCC were negative for CD10. The 16 remaining cases also expressed CD10.

Conclusions: CD10 and RCC are often expressed by clear cell renal cell carcinomas, and they may be useful markers to suggest a renal origin of carcinomas. RCC is less sensitive than CD10. Staining for both of them is usually focal, and thus sensitivity of these techniques decreases when small samples are investigated, such as tissue arrays. The antigen retrieval method is essential for RCC immunohistochemical detection, obtaining the best results with the use of proteolytic enzymes.

Keywords: CD10. CALLA. Renal cell carcinoma marker. Renal cell carcinoma . Clear cell carcinoma. Immunohistochemistry. 
$\mathrm{E}^{1}$ carcinoma renal se caracteriza por la capacidad para producir metástasis en localizaciones muy variadas, que pueden constituir, incluso, su forma de presentación ${ }^{1}$. Aunque este tumor presenta habitualmente una morfología típica de células claras, esta apariencia no es exclusiva de los carcinomas renales ${ }^{2,3}$. Por ello, en ocasiones es necesario un estudio de inmunohistoquímica (IHQ) para confirmar el origen renal de tumores compuestos por células claras, especialmente en biopsias de metástasis cuyo primario es desconocido o dudoso.

$\mathrm{El}$ carcinoma renal de células claras posee un perfil de IHQ característico que consiste en la positividad para citoqueratinas de bajo peso molecular, EMA y vimentina, siendo negativos CEA y citoqueratinas 7 y $20^{4-6}$. Sin embargo este inmunofenotipo no es específico y en los últimos años se ha propuesto el uso de dos anticuerpos, CD10 y el denominado marcador de carcinoma renal (MCR), como indicadores de origen renal de carcinomas $^{7,8}$.

CD10 (CALLA: common acute lymphoblastic leukemia antigen) es una peptidasa de superficie celular descrita inicialmente en determinadas células linfoides y mieloides, por lo que el análisis de su expresión es de utilidad para el diagnóstico de ciertos tipos de linfomas y leucemias $^{9,10}$. Sin embargo, el hallazgo de nuevos tejidos normales y tumorales en los que se expresa CD10 y el desarrollo de anticuerpos monoclonales que funcionan sobre tejido incluido en parafina, han ampliado el uso de este marcador en el diagnóstico anatomopatológico ${ }^{7}$. Entre las nuevas aplicaciones de CD10, destacan el diagnóstico de tumores del estroma endometrial ${ }^{11}$, hepatocarcinomas ${ }^{12,13}$ y carcinoma renal ${ }^{7,14,15}$.

$\mathrm{El}$ anticuerpo monoclonal denominado MCR se ha obtenido frente a una glucoproteína del ribete en cepillo del epitelio del túbulo proxi$\mathrm{mal}^{16}$. Se ha propuesto como un marcador altamente sensible y específico de carcinoma de origen $\mathrm{renal}^{8} \mathrm{y}$ presenta una importante ventaja: su efectividad sobre tejido incluido en parafina, frente a otros que sólo funcionaban en tejido congelado y además eran menos específicos ${ }^{17-20}$.

El uso de matrices tisulares ("tissue arrays") permite investigar diferentes marcadores en series amplias de casos de forma homogénea, con bajo consumo de reactivos $\mathrm{y}$, por tanto, menor $\operatorname{coste}^{21,22}$. Esta técnica consiste en la elaboración de bloques de parafina a partir de cilindros de muy pequeño calibre $(0,6-2 \mathrm{~mm})$ que se extraen, mediante un dispositivo especial o de forma manual, de bloques convencionales, con lo que se reúnen en un solo bloque receptor docenas o cientos de $\operatorname{casos}^{21,23}$.

Hemos analizado la sensibilidad de CD10 y MCR como marcadores de IHQ de carcinoma renal, estudiando la frecuencia y patrón de expresión de ambos en una serie de carcinomas renales de células claras de diferentes grados histológicos.

\section{MATERIAL Y MÉTODOS}

Se elaboraron dos matrices tisulares a partir de 40 bloques de parafina extrayendo dos cilindros de zonas representativas seleccionadas de cada bloque, con aguja de calibre 16G. Los bloques pertenecian al archivo del servicio de patología del hospital POVISA y correspondían a 40 casos de carcinoma renal de células claras $(8 \mathrm{de}$ grado 1, 21 de grado 2,8 de grado 3 y 3 de grado 4 según la clasificación de Fuhrman ${ }^{24}$ ).

Todos los casos habían sido fijados en formol e incluídos en parafina de manera rutinaria. En secciones de 4 micras obtenidas de las matrices tisulares, se realizó técnica de inmunohistoquímica siguiendo el método de estreptavidina marcada con peroxidasa-biotina (LSAB2“, DakoCytomation, Dinamarca). Se utilizaron anticuerpos monoclonales anti CD10 y MCR, ensayándose para este último 4 métodos diferentes de desenmascaramiento antigénico. Los detalles de los anticuerpos utilizados y de la técnica se refieren en la Tabla 1. El cromógeno utilizado fue diaminobencidina. Se incluyeron en las matrices tisulares controles positivos y negativos de la técnica.

Se analizó al microscopio óptico la positividad (tinción marrón) valorándose, como previamente otros autores ${ }^{23}$, la proporción de células reactivas, como: negativo (ninguna célula teñida); positividad muy focal o en células aisladas (+); positividad moderada (++); o extensa reactividad (+++). Para determinar la asociación entre tinción y grado histológico se empleó el test de $\chi^{2}$. Como nivel de significación estadística se consideró un valor de $\mathrm{p} \leq 0.05$. 
Tabla 1

Técnica de inmunohistoquímica: especificaciones

\begin{tabular}{lcccc}
\hline Anticuerpo & $\begin{array}{c}\text { Clon } \\
\text { fabricante }\end{array}$ & Dilución & Incubación & $\begin{array}{c}\text { Recuperación } \\
\text { antigénica }\end{array}$ \\
\hline $\mathrm{CD} 10$ & $\begin{array}{c}56 \mathrm{C} 6, \\
\text { Novocastra } \\
\text { (Reino Unido) }\end{array}$ & $1: 50$ & $\begin{array}{c}30 \text { minutos a } \\
\text { temperatura } \\
\text { ambiente }\end{array}$ & $\begin{array}{c}\text { Proteinasa } \mathrm{K}+\text { olla a } \\
\text { presión con citrato } \\
\text { (5 minutos) }\end{array}$ \\
$\mathrm{MCR}$ & $\begin{array}{c}66.4 . \mathrm{C} 2, \\
\text { Novocastra } \\
\text { (Reino Unido) }\end{array}$ & $1: 10$ & Nocturna a $4^{\circ} \mathrm{C}$ & $\begin{array}{c}\text { Se ensayaron: } \\
\text { *Proteinasa K } \\
\text { *Tripsina }\end{array}$ \\
& & & & *Tripsina + olla \\
& & & & $*$ Tripsina + proteinasa K \\
\hline
\end{tabular}

No se observó asociación estadísticamente significativa entre grado histológico y expresión de CD10 $(\mathrm{p}=0.145)$ o de MCR ( $\mathrm{p}=0.844)$.

\section{DISCUSIÓN}

La inmunorreactividad para CD10 se describió en principio en determinadas neoplasias hematológicas ${ }^{10}$, pero en los últimos años ha ido aumentando el grupo de tejidos conocidos con positividad

\section{RESULTADOS}

Ambos marcadores, CD10 y MCR, tiñeron el epitelio de los túbulos contorneados proximales, que sirvió como control positivo de la técnica.

CD10: Fueron positivos 30 tumores $(75 \%$ de los casos), con variabilidad en la proporción de células teñidas: $12+, 5++$ y $13+++$ (Fig. 1). De los 30 casos positivos, tres correspondían a tumores de grado 1 , diecinueve fueron de grado 2 , cinco de grado 3 y tres de grado 4 (Fig. 2).

MCR: Como método de desenmascaramiento antigénico fue necesario un doble tratamiento enzimático (tripsina + proteasa) habiendo resultado inadecuados los restantes métodos ensayados. Se encontró positividad en 20 casos (50\%): 7 + (Fig. 3), 5 ++ (Fig. 4) y $8+++$. Correspondieron a tres casos de grado 1 , nueve de grado 2 , seis de grado 3 y dos de grado 4 (Fig. 5)

De los 20 casos positivos para MCR, cuatro habían sido negativos para CD10. Los otros 16 expresaban también CD10.

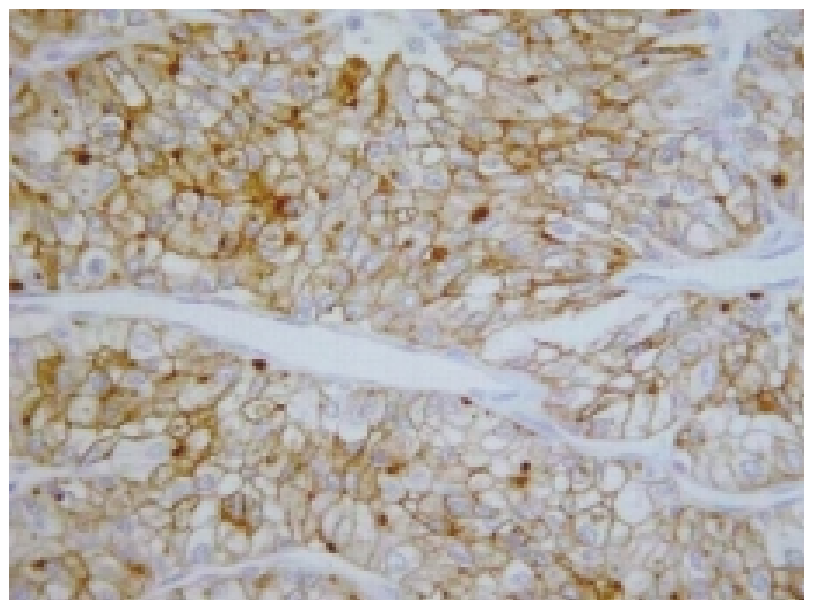

FIGURA 1. Intensa positividad para CD10 en carcinoma renal de células claras. (Inmunoperoxidasa, X400).

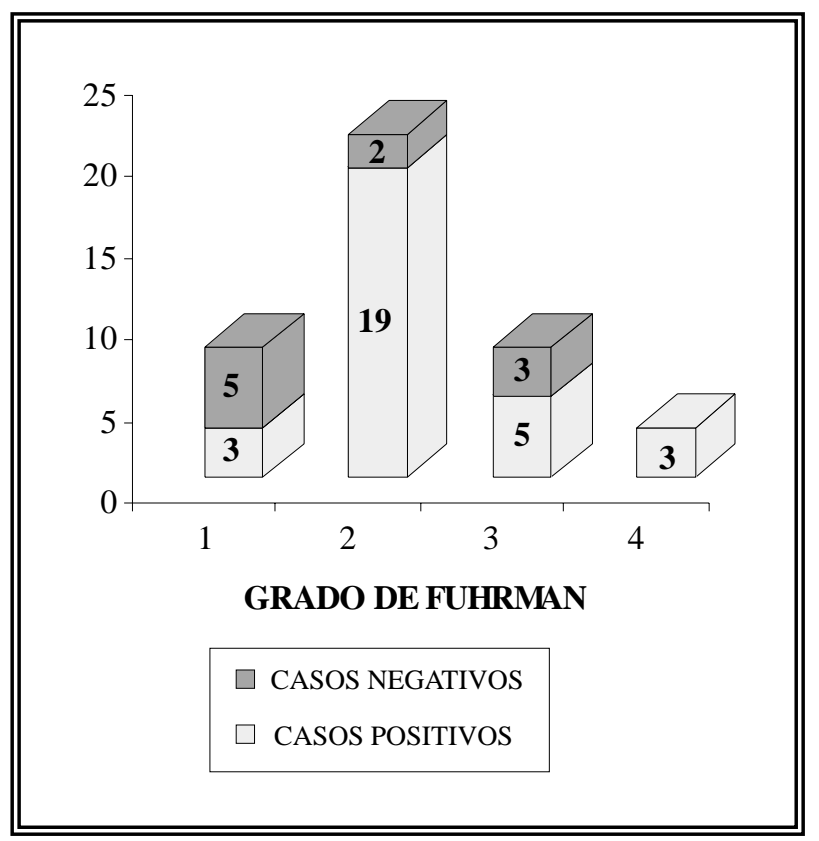

FIGURA 2. Tinción para CD10: distribución de los casos negativos y positivos según grado de Fuhrman.

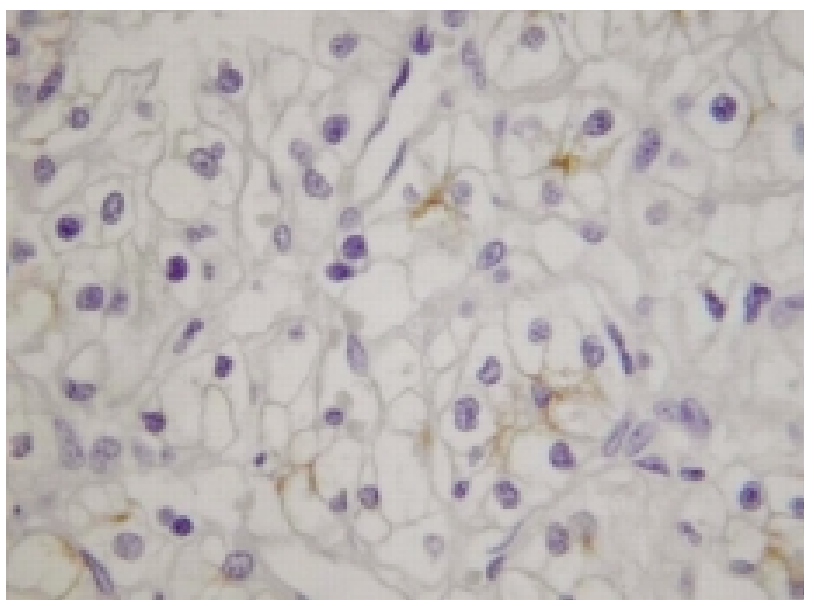

FIGURA 3. Expresión de MCR en células aisladas de carcinoma renal. (Inmunoperoxidasa, X630). 


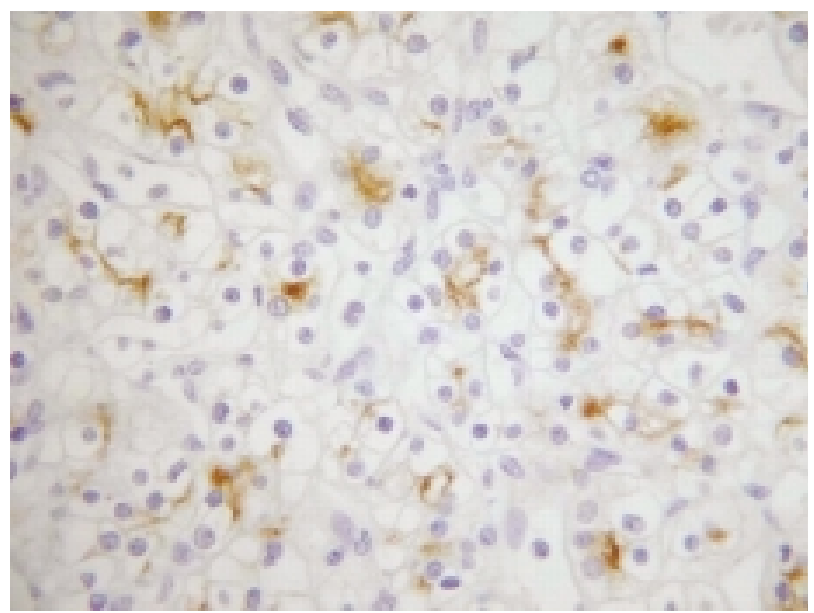

FIGURA 4. Positividad moderada de MCR en carcinoma de células renales. (Inmunoperoxidasa, X400).

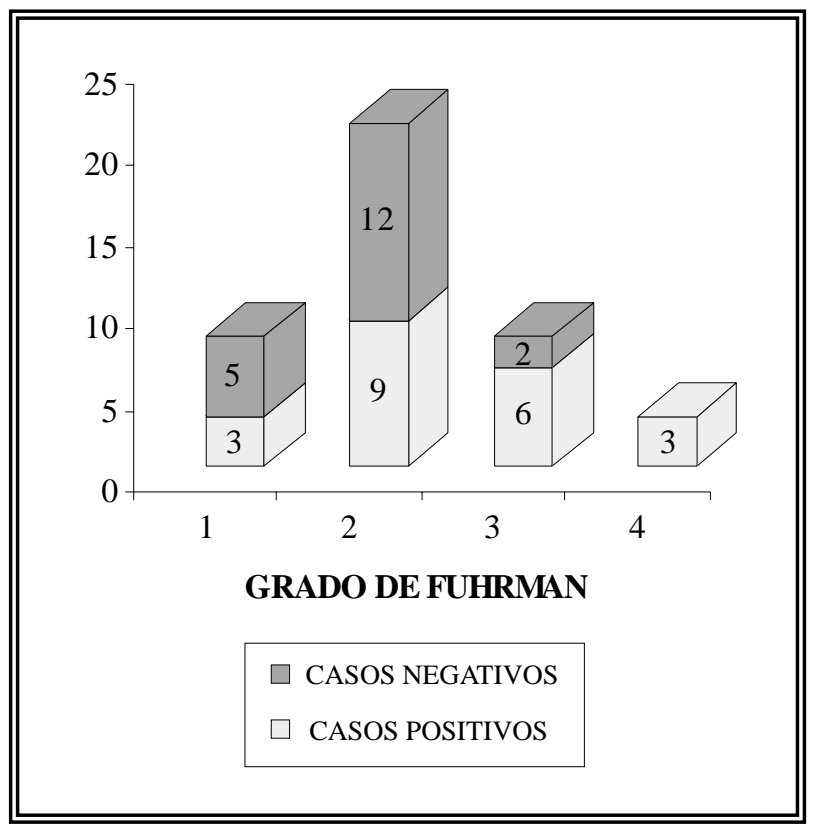

FIGURA 5. Tinción para MCR: distribución de los casos negativos y positivos según grado de Fuhrman.

para dicho marcador. Se ha observado expresión de $\mathrm{CD} 10$ en una variedad de tejidos normales como son células mioepiteliales de mama y parótida $^{7,25}$; metaplasia apocrina de la mama; epitelio glandular intestinal ${ }^{7,25}$; células estromales del endometrio y de la médula ósea ${ }^{7,26}$; canalículos biliares; y neumocitos ${ }^{7,27}$. En el aparato genitourinario se ha descrito en células glomerulares y túbulos contorneados proximales del riñón ${ }^{7,25}$, y en la superficie apical de los ductos prostáticos de calibre grande y ductos epididimarios ${ }^{7,28}$. En consecuencia, el número de neoplasias no hema- tológicas que expresan CD10 se ha ido incrementando, aunque la frecuencia de expresión varía en las diferentes series, que, en ocasiones, incluyen pocos casos. Entre los tumores que presentan positividad para $\mathrm{CD} 10$ con cierta frecuencia, se encuentran melanomas ${ }^{7,29}$, neoplasias digestivas (colon, páncreas, hígado) ${ }^{7,30}$, carcinoma lobulillar de mama, adenocarcinoma y carcinoma de células pequeñas del pulmón, tumores de partes blandas como rabdomiosarcoma, y mesotelioma ${ }^{7}$ . Destacan por presentar muy frecuente expresión, los tumores del estroma endometrial (100\% de los casos positivos) y los del tracto genitourinario, con positividad en tumores de células germinales del testículo (100\% de 19 seminomas, 2 coriocarcinomas y 4 teratomas;y 2 de 3 tumores del saco vitelino estudiados) ${ }^{31}, 54 \%$ de carcinomas uroteliales, $61 \%$ de adenocarcinomas de la próstata y en la mayoría de los carcinomas renales, con una frecuencia que varía entre 82 y $94 \%$ según diferentes series ${ }^{7,14,15,23}$. La expresión de CD10 puede ser útil, además, para diferenciar tipos histológicos de carcinoma renal, y, en concreto, el carcinoma cromófobo ha sido negativo para CD10 en un total de 25 casos estudiados en 2 series diferentes ${ }^{14,15}$, aunque otros autores encuentran positividad en un $28-32 \%$ de los $\operatorname{casos}^{23,32}$. En el carcinoma de ductos colectores los resultados son controvertidos y, en general, las series son muy pequeñas debido a la rareza de esta neoplasia, describiéndose negatividad en 5 casos estudiados ${ }^{15}$, pero observándose positividad en otras series ${ }^{33}$ como en 2 de los 5 casos de Pan et $\mathrm{al}^{23}$. En muestras de PAAF se ha descrito positividad para CD10 en 31 de 41 carcinomas de células renales pertenecientes a dos series ${ }^{2,34}$.

La falta de especificidad de origen renal para el CD10 ha planteado, por tanto, la necesidad de usar otros marcadores de IHQ.

Se ha denominado MCR a un anticuerpo monoclonal desarrollado frente a una fracción del túbulo contorneado proximal ${ }^{16}$. MCR se ha revelado como muy específico de tumor renal, y sólo se ha descrito positividad en otras pocas localizaciones, como mama y paratiroides ${ }^{8,16}$ así como en algunas neoplasias testiculares como carcinoma embrionario $^{16}$ y tumor del saco vitelino (fueron positivos los 8 casos estudiados por Yan et al, que incluso proponen una posible aplicación de MCR para el diag- 
nóstico de este tumor) ${ }^{35}$, así como raramente en carcinomas de células claras del ovario (positividad focal en 2 casos de 14 estudiados) ${ }^{3}$.

La tinción para MCR en el carcinoma renal suele ser focal y se han considerado como positivos, casos con porcentajes bajos de células inmunorreactivas ${ }^{36}$. La sensibilidad de MCR en el carcinoma renal de células claras es elevada habiéndose comunicado positividad en un $65-100 \%$ en las series que estudian secciones convencionales de parafina ${ }^{3,8,14,16}$. Considerando en conjunto estas series, la positividad aparece en 150 de un total de 183 casos lo que supone un $81,9 \% \%^{3,8,14,16}$. Se ha descrito con menor frecuencia la reactividad para MCR en tumores metastásicos al compararla con la de los tumores primarios: $84 \%$ vs. $93 \%$ respectivamente en la serie de Yoshida e $\operatorname{Imam}^{16}$, y en la de McGregor et al. $67 \%$ vs. $84 \%$ respectivamente ${ }^{8}$.

En nuestra casuística hemos encontrado, sin embargo, unos porcentajes de positividad para ambos marcadores menores que los de otras series: $75 \%$ para CD10 y 50\% para MCR Nuestros resultados son muy similares a los de Pan y cols recientemente publicados, que observaron en carcinomas renales de células claras unos porcentajes de positividad del $82 \%$ para CD10 y $47 \%$ para $\mathrm{MCR}^{23}$. Esta serie y la nuestra tienen en común un aspecto metodológico relevante como es el haberse realizado ambas con matrices tisulares, con lo que la representatividad del tumor no es equivalente a la de las secciones convencionales lo que explicaría el menor número de casos positivos observado. Ello parece corroborado por el hecho de que, como hemos señalado, en general los casos positivos no muestran una tinción generalizada sino sólo en un porcentaje de células. En este sentido, observamos también menor positividad para MCR en dos series publicadas de muestras citológicas de PAAF de carcinomas renales primarios y metastásicos, con porcentajes de $33.3 \%$ sobre 12 casos estudiados y $38 \%$ de un total de $21 \operatorname{casos}^{34,36}$. Una conclusión práctica que se deriva de nuestros resultados y de los de estas series, es que en muestras de pequeño tamaño de carcinomas renales (por ejemplo biopsias por aguja o muestras citológicas) no debemos esperar encontrar expresión de CD10 y MCR con la frecuencia que se describe en gran parte de la bibliografía publicada ${ }^{3,8,16}$.
Por otra parte, hay que señalar la posibilidad de que existan tumores que siendo negativos para CD10 expresen MCR, como ocurrió en 4 de nuestros casos (20\% de los MCR positivos), por lo que en la práctica sería aconsejable usar ambos marcadores para el estudio de un caso concreto.

Además de ser indicadores de origen renal en los carcinomas de células claras, MCR y CD10 pueden ser útiles para diferenciar dicha neoplasia de otros tipos de tumor renal como el carcinoma cromófobo y el oncocitoma que suelen ser negativos para ambos marcadores aunque no de forma constante $\mathrm{e}^{8,14,23,33}$.

En nuestra experiencia un aspecto fundamental para obtener la máxima sensibilidad de la técnica IHQ para MCR ha sido el método de desenmascaramiento antigénico elegido, como también observaron otros autores ${ }^{8}$. En la mayor parte de las series, incluida la nuestra, se han utilizado enzimas proteolíticos, en uno o varios pa$\operatorname{sos}^{8,14,16,23,34}$ siendo menos habitual el uso de calor $^{3,35,36}$.

En conclusión, el estudio de IHQ de CD10 y MCR puede ser útil en el estudio histológico de carcinomas: aunque un resultado negativo no excluye carcinoma renal, la positividad para alguno de estos marcadores apoya dicho diagnóstico.

Agradecimiento. Agradecemos a las Sras. Ana Rosa Calderón Fernández y Carmen Rodríguez Otero, bibliotecarias del Hospital POVISA, su colaboración en la realización de este trabajo.

\section{REFERENCIAS}

1. Murphy WM, Beckwith JB, Farrow GM. Atlas of tumor pathology. Tumors of the kidney, bladder, and related urinary structures. Armed Forces Institute of Pathology 1994: 92131.

2. Yang B, Ali SZ Rosenthal DL. CD10 facilitates the diagnosis of metastatic renal cell carcinoma from primary adrenal cortical neoplasm in adrenal fine-needle aspiration. Diagn Cytopathol 2002;27:149-152.

3. Cameron RI, Ashe P, O'Rourke DM, Foster H, Mccluggage. A panel of immunohistochemical stains assists in the distinction between ovarian and renal clear cell carcinoma. Int $\mathrm{J}$ Gynecol Pathol 2003;22:272-276.

4. Wang NP, Zee S, Zarbo RJ, Bacchi CE, Gown AM. Coordinate expression of cytokeratins 7 and 20 defines unique subsets of carcinomas. Appl Immunohistochem Mol Morphol 1995;3(2):99-107.

5. Chu P, WU E, Weiss LM. Cytokeratin 7 and Cytokeratin 20 expression in epithelial neoplasms: a survey of 435 cases. Mod Pathol 2000;13(9):962-972. 
6. Eble JN, Sauter G, Epstein JI, Sesterhenn IA. World Health Organization Classification of Tumours. Pathology and genetics of tumours of the urinary system and male genital organs. International Agency for Research on Cancer Press 2004:23-25.

7. Chu P, Arber DA. Paraffin-section detection of CD10 in 505 nonhematopoietic neoplasms; frequent expression in renal cell carcinoma and endometrial stromal sarcoma. Am J Clin Pathol. 2000;113(3):374-82.

8. McGregor DK, Khurana KK, Cao C, Tsao CC, Ayala G, Krishnan B, Ro JY, Lechago J, Truong LD. Diagnosing primary and metastatic renal cell carcinoma; the use of the monoclonal antibody renal cell carcinoma marker. Am J Surg Pathol. 2001;25(12):1485-1492.

9. Gafford JT, Skidgel RA, Erdos EG, Hersh LB. Human kidney "enkephalinase", a neutral metalloendopeptidase that cleaves active peptides. Biochemistry. 1983 Jun 21;22(13): 3265-3271.

10. Arber DA, Weiss LM: CD10. A review. Appl Immunohistochem Mol Morphol 1997;5:125-140.

11. Mccluggage WG, Sumathi WP, Maxwell P. CD10 is a sensitive and diagnostically useful immunohistochemical marker of normal endometrial stroma and of endometrial stromal neoplasms. Histopathology 2001;39(3):273-278.

12. Lin F, Abdallah H. Diagnostic utility of CD10 in discriminating hepatocellular carcinoma from metastatic carcinoma in FNAB of the liver. Lab Invest 2003;83(1):73A.

13. Luckasevic TM, Sadd RS, Noga CM, et al. Diagnostic value of CD10 and CD34 expressions in separating hepatocellular carcinoma from metastatic carcinoma in fine needle aspiration cytology. Lab Invest 2003;83(1):74A.

14. Avery AK, Beckstead J, Renshaw AA, Corless CL. Use of antibodies to RCC and CD10 in the differential diagnosis of renal neoplasms. Am J Surg Pathol 2000; 24(2): 203210 .

15. Kim MK, Kim S. Immunohistochemical profile of common epithelial neoplasms arising in the kidney. Appl Immunohistochem Mol Morphol 2002; 10(4): 332-338.

16. Yoshida SO, Imam A. Monoclonal antibody to a proximal nephrogenic renal antigen: immunohistochemical analysis of formalin-fixed, paraffin-embedded human renal cell carcinomas. Cancer Res 1989;49(7):1802-1809.

17. Finstad CL, Cordon-Cardo C, Bander NH, Whitmore WF, Melamed MR, Old LJ. Specificity analysis of mouse monoclonal antibodies defining cell surface antigens of human renal cancer. Proc Natl Acad Sci USA 1985;82(9):29552959.

18. Luner SJ, Ghose T, Chatterjee S, Cruz HN, Belitsky P. Monoclonal antibodies to kidney and tumor-associated surface antigens of human renal cell carcinoma. Cancer Res 1986; 46(11):5816-5820.

19. Oosterwijk E, Ruiter DJ, Wakka JC, Huiskens-van der Meij JW, Jonas U, Fleuren GJ, Zwartendijk J, Hoedemaeker P, Warnaar SO. Immunohistochemical analysis of monoclonal antibodies to renal antigens: application in the diagnosis of renal cell carcinoma. Am J Pathol 1986: 123(2):301309.

20. Scharfe T, Becht E, Kaltwasser R, Thuroff JW, Jacobi GH, Hohenfellner R. Tumor-specific monoclonal antibodies for renal cell carcinoma. Eur Urol 1985; 11(2):117-120.

21. Nocito A, Kononen J, Kallioniemi OP, Sauter G. Tissue microarrays (TMAs) for high-throughput molecular pathology research. Int J Cancer 2001;94(1):1-5.
22. Gelabert-Mas A, Rioja-Sanz LA. El microarray tisular (TMA): una nueva y poderosa herramienta para la investigación clínico-molecular al servicio de la urología. Actas Urol Esp 2003; 27(1):1-2.

23. Pan CC, Chen PC, Ho DM. The diagnostic utility of MOC31, BerEP4, RCC marker and CD10 in the classification of renal cell carcinoma and renal oncocytoma: an immunohistochemical analysis of 328 cases. Histopathology 2004; 45(5): 452-459.

24. Fuhrman SA, Lasky LC, Limas C. Prognostic significance of morphologic parameters in renal cell carcinoma. Am J Surg Pathol 1982; 6(7): 655-663

25. Metzgar RS, Borowitz MJ, Jones NH, Dowell BL. Distribution of common acute lymphoblastic leukemia antigen in nonhematopoietic tissues. J Exp Med 1981; 154(4): 1249-1254.

26. Keating A, Whalen CK, Singer JW. Cultured marrow stromal cells express common acute lymphoblastic leukaemia antigen (CALLA): implications for marrow transplantation. Br J Haematol. 1983 55(4):623-628.

27. Johnson AR, Ashton J, Schulz WW, Erdos EG. Neutral metalloendopeptidase in human lung tissue and cultured cells. Am Rev Respir Dis 1985;132(3):564-568.

28. Erdos EG, Schulz WW, Gafford JT, Defendini R. Neutral metalloendopeptidase in human male genital tract: comparison to angiotensin I-converting enzyme. Lab Invest 1985; 52:(4):437-447.

29. Carrel S, Zografos L, Schreyer M, Rimoldi D. Expression of CALLA/CD10 on human melanoma cells. Melanoma Res 1993; 3(5):319-323.

30. Sato Y, Itoh F, Hinoda Y, Ohe Y, Nakagawa N, Ueda R, Yachi A, Imai K. Expression of CD10/neutral endopeptidase in normal and malignant tissues of the human stomach and colon. J Gastroenterol 1996;31(1):12-17.

31. Bacon CM, Vo L, Parkinson MC, Freeman A. Expression of CD10 in testicular tumours. Mod Pathol 2004;17(Supl.1): 139A.

32. Bismar TA, Che M, Sakr WA, Grignon DJ. The value of immunohistochemistry in the diagnosis of epithelial renal neoplasms with pink cytoplasm. Lab Invest 2003;83(1):142A.

33. Radhakrishnan A, Lim SD, Patel A, Hennigar B, Shehata B, Amin MB. Utility of a contemporary immunohistochemical panel in the differential diagnosis of adult renal epithelial neoplasms: a study in tissue microarrays to simulate experience in renal biopsy specimens. Mod Pathol 2004;17(Supl.1):173A.

34. Cangiarella J, Wei XJ, Simsir A, Yee H, Chhieng D. Comparison of CD10 and renal cell carcinoma marker in diagnosing primary and metastatic renal cell carcinoma on fine needle aspiration biopsy. Lab Invest 2003;83(1):61A.

35. Yan M, Ghorab Z, Nadji M. Renal cell carcinoma antigen is expressed by yolk sac tumors and yolk sac elements of embryonal carcinomas. Appl Immunohistochem Mol Morphol 2003;11(2):113-115.

36. Gokden N, Mukunyadzi P, James JD, Gokden M. Diagnostic utility of renal cell carcinoma marker in cytopathology. Appl Immunohistochem Mol Morphol 2003;11(2):116-119.

Dr. J.A. Ortiz Rey

Servicio de Patología. Hospital POVISA.

Salamanca, 5 - 36211 Vigo (Pontevedra)

E-mail: jaortiz@povisa.es

(Trabajo recibido el 21 de septiembre de 2005) 